\title{
Caracterización Geomorfológica como Propuesta para la Planificación Ambiental y Territorial. Un Ejemplo de Aplicación en Brasil con Perspectiva de Aplicación en Costa Rica
}

\author{
A Geomorphological Characterization as a Proposal for \\ Environmental and Territorial Planning. An Example of \\ Application in Brazil Intended to Be Replicated in Costa Rica
}

Max Furrier ${ }^{l}$

Universidade Federal da Paraíba, Brasil

http://dx.doi.org/10.15359/rgac.61-2.8

\begin{abstract}
RESUMEN
Este trabajo contempla una caracterización geomorfológica como base para la planificación ambiental y territorial en una región costera de Brasil. Desde el punto de vista geomorfológico, el área de estudio se encuentra ubicada sobre las mesetas y tierras bajas costeras, donde predomina la Formación Barreiras y sedimentos del Cuaternario. Esta área posee una grande diversidad pedológica, con diversos usos y ocupaciones. La integración de los datos obtenidos, generados, analizados y discutidos, fueron sintetizados en la plancha de fragilidad de los ambientes naturales y antrópicos que permite visualizar cómo los componentes del medio físico se integran y pueden influenciar en las dinámicas socio-económicas. Esta integración de datos es de fundamental importancia en la planificación ambiental y territorial, principalmente en países en desarrollo como Brasil y Costa Rica, donde en muchas regiones hay pocos datos o los datos son dispersos.
\end{abstract}

Palabras clave: cartografía geomorfológica, mesetas costeras, plancha de fragilidad.

1 Profesor Doctor de la Universidade Federal da Paraíba, Brasil. Correo electrónico: max.furrier@gmail.com

Fecha de recepción: 10 de enero de 2018

Fecha de aceptación: 31 de enero de 2017 
Max Furrier. A Geomorphological Characterization as a Proposal for Environmental and Territorial Planning. An Example of Application in Brazil Intended to Be Replicated in Costa Rica

\begin{abstract}
This work contemplates a geomorphological characterization as a basis for environmental and territorial planning in a coastal region of Brazil. From the geomorphological point of view, the study area is located on the plateaus and coastal lowlands, where the Barreiras Formation and Quaternary sediments predominate. This area has a great pedological diversity, with different uses and occupations. The generated, analyzed and discussed data were synthesized using the fragility map of natural and anthropogenic environments; the map allows visualizing how the components of the physical environment are integrated and can influence the socio-economic dynamics. This data integration is of fundamental importance in environmental and territorial planning, mainly in developing countries such as Brazil and Costa Rica, where in many regions there are little data or data are scattered.
\end{abstract}

Keywords: geomorphological mapping, coastal tableland, fragility map.

\title{
Introducción
}

Las zonas costeras ejercen gran atracción y fascinación, de tal forma que las poblaciones humanas tienden a aumentar su ocupación. Cuando ésta se incrementa, los factores antrópicos se superponen a los fenómenos dinámicos de la naturaleza, agravando las susceptibilidades naturales y la introducción de susceptibilidades inducidas, creando complejas situaciones de crisis de diversos tipos (Suguio et al., 2005). Debido a la complejidad natural y al nivel de intervención humana, la organización del espacio de ambientes costeros y adyacencias propone mayor atención a estas zonas de relieve para el mantenimiento de su equilibrio, manifestando la necesidad de un mayor conocimiento de sus estructuras y de las fuerzas que intervienen en el ajuste de sus formas (Feitosa, 1996).

Las mesetas costeras en zonas litorales, como todo ambiente natural cuando queda libre de intervenciones humanas, presentan desde el punto de vista geomorfológico, características de ambientes de inestabilidad potencial, siendo susceptibles a procesos de origen marino, atmosféricos, endógenos (tectonismo, geoestasia), biológicos y fluviales. Desde el momento en que cualquier ambiente natural comienza la etapa de actividades humanas, se encontrará fuera del equilibrio de los procesos naturales actuantes, tornándose en un ambiente de inestabilidad emergente (Ross, 1994).

Los estudios sobre las zonas costeras y adyacentes vienen aumentando considerablemente en los últimos años, y gradualmente adquieren gran importancia en la planificación ambiental y territorial, principalmente, en áreas donde la intervención humana provoca la destrucción de algún patrimonio o interfiere de modo drástico en los ecosistemas (Ab'Saber; 2000; Hoefel, 
Max Furrier. Caracterización Geomorfológica como Propuesta para la Planificación Ambiental y Territorial. Un Ejemplo de Aplicación en Brasil con Perspectiva de Aplicación en Costa Rica

1998; Mesquita, 2003; Muehe, 1994). De acuerdo con estas necesidades, se propone un estudio integrado del sur de la costa del Estado de Paraíba y sus áreas adyacentes, en el cual el relieve es la base de la investigación, y de esta forma intentar caracterizar este relieve y sus relaciones con la geología, suelos, clima, vegetación y el uso y ocupación del suelo.

Esta integración de datos, desde los datos litológicos/geológicos, pasando por la interpretación de los mismos y la formulación de una plancha geomorfológica con datos de los suelos y del uso y ocupación de la tierra no es una terea fácil, principalmente en regiones con un nivel de desarro1lo bajo, como sucede en gran parte de Brasil y Costa Rica. Muchos datos están dispersos en servicios geológicos, en servicios geográficos, institutos agrícolas y ambientales. Encontrarlos y utilizar los necesarios para un estudio integrado es una tarea dura, sin embargo, de extrema importancia para esta propuesta holística.

El relieve es escenario de las más variadas actividades humanas. Cada una de las geoformas del relieve, debido a su génesis y a su proceso evolutivo, ofrece a la población en ellas ubicadas, una variedad de beneficios y riesgos, que pueden ser transformados, de acuerdo con el tipo de intervención impuesta por el hombre (Marques, 1994).

La estabilidad del medio físico está íntimamente ligada a la actividad humana y a su grado de intervención. A diferencia de algunas regiones del planeta en las que no se desarrollan actividades humanas como en la Antártida, los ambientes naturales se presentan en un estado de equilibrio dinámico, ofreciendo una mayor o menor fragilidad en función de sus características físicas y morfogenéticas. Desde el momento en que el ser humano empieza a actuar en estos ambientes, el equilibrio dinámico es modificado, trayendo como consecuencia condiciones de fragilidad que son medidas por el grado de intervención humana (Ross, 1996).

El objetivo de la planificación ambiental, según los principios de Santos (2004) es establecer normas para territorios complejos y, para ello, él necesita estar suficientemente ligado a la realidad en sus múltiples aspectos. Tiene que interpretar el medio en relación con su composición. Por esta razón, su diagnóstico busca comprender el medio de forma global, a través del levantamiento de datos y su integración. Muchas veces, hay datos producidos por el servicio geológico que pueden ser útiles a la planificación desde que estos son comprendidos para tales fines. 
Para estudios integrados del paisaje, los datos de geomorfología se consideran imprescindibles. El análisis del relieve y el mapeo permiten sintetizar la historia de las interacciones dinámicas que ocurren entre el sustrato litológico, la tectónica y las variaciones climáticas (Ross, 1990). El relieve no es solo producto de los procesos superficiales. Tiene una historia geológica que no puede ser renegada.

Por medio del presente trabajo, se busca la suma y la integración de datos físicos y sociales, intentando mostrar el estado actual, proporcionando la integración de los mismos para que puedan servir de base en futuros proyectos de planeación ambiental y territorial, como también, en futuros soportes metodológicos con el intento de su aplicación en Costa Rica.

\section{Localización del área de estudio}

El área de estudio está localizada en la parte centro sur del litoral del Estado de Paraíba (Brasil), comprendiendo un área ubicada en las cartas topográficas de Conde y Jacumã, a escala 1:25.000. Esta área está delimitada al norte, por el paralelo $7^{\circ} 15^{\prime} 00^{\prime \prime}$; al sur, por el paralelo $7^{\circ} 22^{\prime} 30^{\prime \prime}$; al oeste con el meridiano $35^{\circ} 00^{\prime} \mathrm{W}$; y al este, con el Océano Atlántico (Figura 1). 
Max Furrier. Caracterización Geomorfológica como Propuesta para la Planificación Ambiental y Territorial. Un Ejemplo de Aplicación en Brasil con Perspectiva de Aplicación en Costa Rica

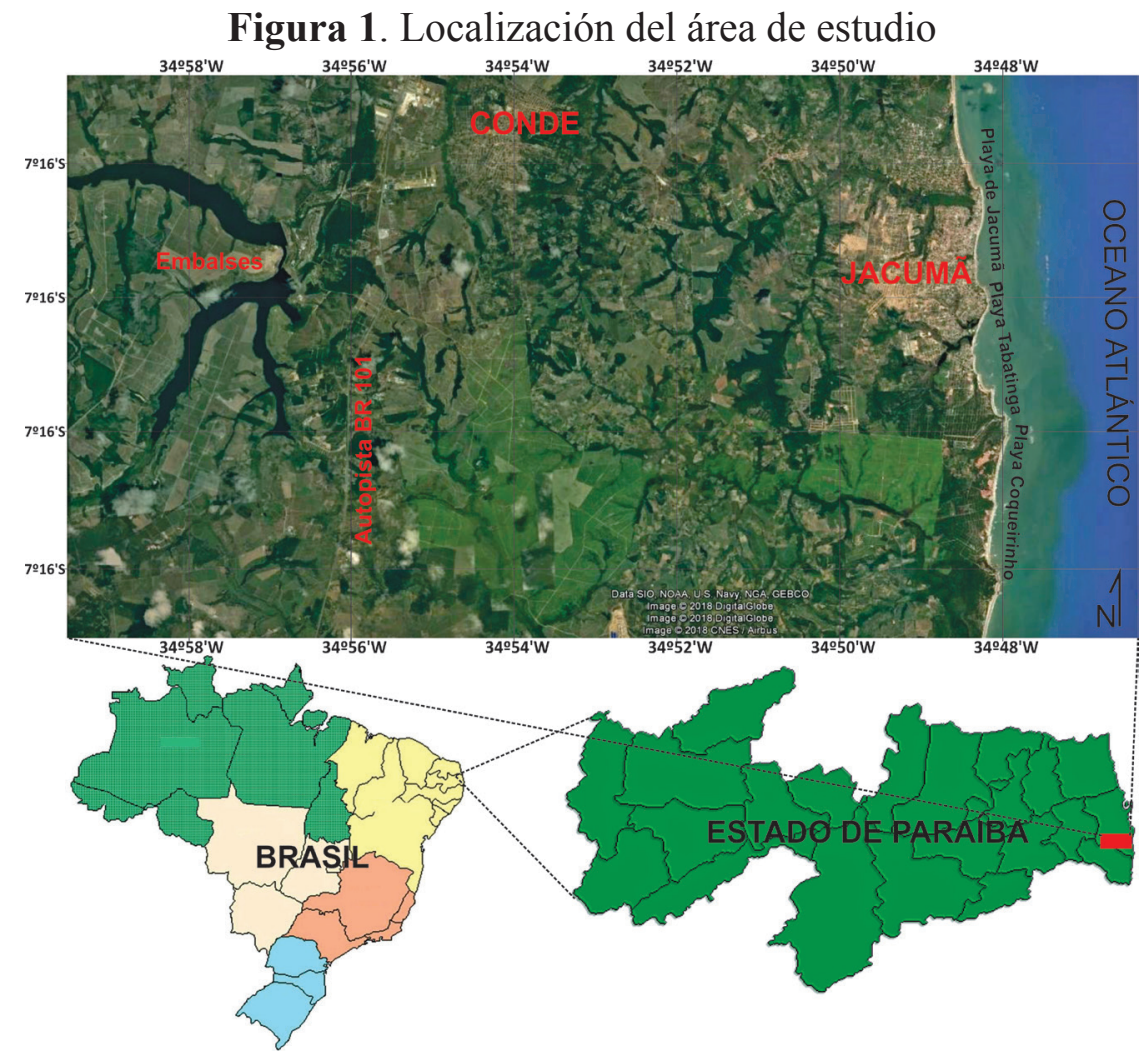

Fuente: Adaptado de Google Earth, 2017.

\section{Características naturales}

\subsection{Geología}

La zona se emplaza en la Cuenca Sedimentaria Paraíba, la última parte de la plataforma sudamericana separada del continente africano, durante la apertura del Océano Atlántico (Françolin y Szatmari, 1987). La Cuenca Paraíba está clásicamente dividida en subcuencas y el área de estudio se encuentra localizada en la Subcuenca Alhandra.

Sobre la Subcuenca Alhandra se depositó la Formación Barreiras del Mioceno. Esta formación superficial la cubre totalmente, avanzando hacia el oeste, donde cubre parte del basamento cristalino Proterozoico. En el sector extremo oeste del área de estudio, la Formación Barreiras reposa directamente sobre el basamento cristalino precámbrico, y extendiéndose 
hacia el Este; esta formación cubre discordantemente las formaciones geológicas cretácicas de la Cuenca Paraíba hasta alcanzar planicies costeras o terminar en acantilados.

El espesor de la Formación Barreiras en el Estado de Paraíba es bastante variable, alcanzando máximos entre 70 y 80 m (Leal e Sá, 1998). Los diferentes espesores actualmente verificados son explicados recientemente con los estudios de la tectónica regional cenozoica, originada por las reactivaciones de las antiguas fallas del basamento cristalino del Proterozoico, debido al constante movimiento de la Placa Sudamericana (Brito Neves et al., 2004).

\section{Depósitos cuaternarios}

Los depósitos cuaternarios cubren una fracción considerable del área de estudio. Estos depósitos pueden ser divididos en dos grandes unidades: depósitos marinos/transicionales y depósitos continentales. Los depósitos marinos/transicionales identificados son los siguientes: terrazas marinas del Pleistoceno, terrazas marinas del Holoceno, arrecifes rocosos, depósitos de mangle y arenas de playa; y los depósitos continentales identificados son: depósitos coluviales, conos de deyección, dunas inactivas y depósitos aluviales.

\subsection{Geomorfología}

El área de estudio está incluida en dos dominios geomorfológicos generales y distintos, cada uno comprende varias unidades geomorfológicas. Los dominios reciben las siguientes denominaciones locales: Mesetas Costeras Bajas y Tierras Bajas Costeras.

\section{Mesetas Costeras Bajas}

Las Mesetas Costeras Bajas se encuentran en la macro-compartimentación de las mesetas costeras brasileñas. Este compartimiento geomorfológico es sostenido, predominantemente, por la Formación Barreiras. En general, constituyen unidades geomorfológicas de superficies aplanadas y suavemente inclinadas hacia el este, siendo abruptamente interrumpidas por las incisiones fluviales y por los acantilados marinos esculpidos por la abrasión marina actual y/o pretérita. 
Max Furrier. Caracterización Geomorfológica como Propuesta para la Planificación Ambiental y Territorial. Un Ejemplo de Aplicación en Brasil con Perspectiva de Aplicación en Costa Rica

La red de drenaje del área de estudio sufre un fuerte control estructural. Las fracturas desencadenadas por los movimientos tectónicos fueron aprovechadas por los procesos lineales de disección con la profundización de los cauces de los cursos de agua. En el área de estudio, se observa un intenso trabajo de disección fluvial responsable de exhumar las formaciones del Grupo Paraíba, generando grandes anfiteatros, algunos bien amplios y otros más encajados. Se percibe cierta alineación de la disección provocada por la erosión regresiva y lineal, aprovechando nítidamente las líneas de fracturas y fallas.

Aunque el tectonismo cada vez explica más la intensificación de los procesos denudacionales que ayudan a esculpir las mesetas costeras, no se pueden dejar atrás los procesos morfoclimáticos que actúan y seguirán actuando de forma intensa, modelando el relieve local. Una estructura describe las características generales del área y los procesos morfoclimáticos que modelan el relieve. Las formas son productos tanto del tectonismo como de los procesos morfoclimáticos pasados y actuales; por lo tanto, es imposible separarlos o incluso medirlos independientemente.

\section{Tierras Bajas Costeras}

Son terrenos relativamente planos de baja altitud, formados por sedimentos depositados durante el Cuaternario. Tienen altitudes modestas, generalmente inferiores a $10 \mathrm{~m}$, aunque se pueden presentar altitudes superiores en planicies fluviales más lejanas a la línea de costa. Las Tierras Bajas Costeras están compuestas por diferentes formas que resultan de la acumulación de sedimentos marinos, fluviales y fluvio-marinos. Sus características geomorfológicas se dividen en playas, terrazas y planicies marinas, planicies fluvio-marinas, y planicies y terrazas fluviales.

\subsection{Pedología}

Debido al clima cálido y húmedo de la región, la mayoría de los suelos presentes en el área, evidencian abundancia de minerales secundarios, principalmente, óxidos de hierro e hidróxido de aluminio, pobres en cationes básicos, sobre todo calcio, magnesio y potasio. Las lluvias, presentes en todos los meses del año, son bastantes concentradas en otoño e invierno, haciendo que grandes volúmenes de agua se infiltren, lixiviando hacia el nivel freático muchos nutrientes del suelo (Lepsch, 2002). 
De acuerdo con Paraíba (2004), el área de estudio tiene ocho tipos principales de suelos, todos con baja fertilidad natural, gran concentración de granos de cuarzo y elevada acidez. Estas características físicas y texturas confieren a los suelos una baja resistencia a la erosión, cuando los mismos se encuentran en pendientes con inclinación elevada. La caracterización pedológica del área de estudio es necesaria para la elaboración de la carta de fragilidad de los ambientes naturales y antrópicos, siguiendo la metodología propuesta por Ross (1994), cuyo componente suelo es jerarquizado con respecto a su potencial de erosión.

La distribución de los suelos en el área de estudio está íntimamente relacionada con el relieve local y con la litología. Sobre las mesetas desarrolladas sobre la Formación Barreiras predominan los Argissolos Rojos-Amarillos, muchas veces asociados a los Latosoles Rojos-Amarillos y a los Espodosoles; en las planicies fluviales se desarrollan los Neosoles Flúvicos y Gleysoles; en las planicies fluvio-marinas son encontrados los Suelos de Manglar, y en las planicies marinas se presentan los Neossolos Quatzarénicos.

\subsection{Clima}

De acuerdo con la clasificación climática de Köppen, que toma como base principal los criterios de temperatura y distribución estacional de precipitación, el área de estudio se encuentra bajo el dominio del clima Tropical Lluvioso, con temporada con menos lluvia en el verano. La ausencia de periodos fríos (temperatura $>18^{\circ} \mathrm{C}$ ) es otra característica excepcional de este tipo de clima. El área de estudio presenta gran homogeneidad estacional y espacial de temperatura, que es elevada durante prácticamente todo el año con una media anual de $25,6^{\circ} \mathrm{C}$. La pluviosidad media anual es de $1.750 \mathrm{~mm}$, siendo el mes de junio el más lluvioso, con pluviosidad media de 300 mm, y el mes de noviembre el más seco con 30 mm (Brasil, 1990).

\subsection{Vegetación y uso de la tierra}

El área de estudio presenta una variada vegetación que refleja las diferentes condiciones ambientales en sus diversos compartimientos morfopedológicos. También se evidencia la fuerte acción antrópica sobre todos los tipos de vegetación del área, incluso en algunas zonas las alteraciones son irreversibles, debido a los cortes completos de la madera para 
Max Furrier. Caracterización Geomorfológica como Propuesta para la Planificación Ambiental y Territorial. Un Ejemplo de Aplicación en Brasil con Perspectiva de Aplicación en Costa Rica

obtención de leña y también para la agroindustria de la caña de azúcar (Etanol), la agricultura de subsistencia y la expansión urbana.

A finales de los años de 1960, el cultivo de caña de azúcar se limitaba a las terrazas y planicies fluviales de los ríos de la región y a algunas zonas con pendientes menos inclinadas en las mesetas. En las partes superiores de las mesetas las condiciones edáficas eran desfavorables (suelos distróficos y arenosos) y, por lo tanto, estaban destinados a los cultivos de subsistencia (Moreira y Targino, 1997). La creación de PROALCOOL, en 1975, apoyado por una fuerte política de incentivos fiscales y financieros, permitió la expansión de la caña de azúcar en las mesetas costeras, rompiendo con la barrera natural impuesta por las condiciones edáficas desfavorables.

El manglar representa la formación más homogénea y uniforme del área de estudio. Este tipo de vegetación se establece en depósitos arcilloarenosos, ricos en materia orgánica en descomposición, y sujetos a flujos y reflujos de las mareas. Por lo tanto, es un tipo de vegetación adaptada a la salinidad y escases de oxígeno, que es consumido en el proceso de descomposición de la materia orgánica en durante la deposición constante.

En las fuertes pendientes de los acantilados inactivos o en los depósitos coluviales de sus bases, se encuentra otro tipo de vegetación denominada como Pionera de Acantilado, que es una especie herbácea y arbustiva, que se presenta sobre los sedimentos areno-arcillosos de la Formación Barreiras. Esta especie es un importante indicador de inactividad erosiva intensa y reciente, por consiguiente, su estudio es fundamental para diferenciar los acantilados inactivos de los acantilados activos.

En el área de estudio se encuentra el municipio de Conde, con una población residente de 23.975 habitantes (Brasil, 2016). La ciudad sede está ubicada en el centro del área (Figura 1), pero este municipio posee un distrito costero llamado Jacumã, donde la población es muy variable, debido a la estacionalidad, multiplicando su población en las vacaciones de verano. Además, en toda el área costera se encuentra una forma de ocupación humana como: resorts, condominios, etc.

\section{Fundamentos teórico-metodológicos}

Con la preocupación de dar al presente trabajo una estructura rigurosa, en la cual se establezca sistemáticamente un comienzo, un medio y un fin, fue incorporada la propuesta metodológica elaborada 
por Libault (1971), que es genérica y aplicable a los diferentes temas de una investigación.

Según Libault (1971), para el desarrollo metodológico se tienen en cuenta cuatro niveles: el nivel compilatorio o característico, que tiene por objetivo recolectar datos; un nivel correlativo, en donde se realizan comparaciones de la información; un nivel semántico, en el cual se realiza la interpretación de los datos seleccionados o correlacionados; y finalmente, un nivel normativo, en donde los resultados se traducen en normas apropiadas a la realidad, la prospectiva y la planeación. Aquí es importante aclarar que solamente una compilación de datos no es una investigación científica, es necesario una correcta integración y correlación entre los mismos, mientras que la adquisición de datos es de extrema importancia.

A principios de los años 1980, la conservación y la preservación de los recursos naturales y el papel del hombre integrado en el medio pasaron a tener una función muy importante en la discusión de la calidad de vida, surgiendo entonces la tendencia de elaborar planificaciones regionales con elementos provenientes del medio natural y antropizado analizados de forma interactiva. Independiente de los objetivos o del lugar planeado, esa estrategia exigía la espacialización de un conjunto amplio de datos que necesitaban ser comparados, superpuestos y evaluados de manera holística (Santos, 2004). Esto se torna aún más importante para áreas extremadamente complejas y deficientes de información, como en muchos sitios de Brasil y Costa Rica.

La planificación es un proceso continuo que involucra la recolección, organización y análisis sistematizados de las informaciones, por medio de procedimientos y métodos, para llegar a decisiones o elecciones acerca de las mejores alternativas para el aprovechamiento de los recursos disponibles. Así, como planear implica identificar, seleccionar y destinar recursos, para que eso sea hecho de manera adecuada es necesario ubicarlos y conocerlos en cantidad y calidad, así como tener claridad sobre los objetivos para los cuales serán dirigidos. En suma, es necesaria alguna forma de espacialización de los recursos presentados por el diagnóstico, así como el conocimiento de los conflictos derivados de su conservación y uso (Slocombe, 1993). 
Max Furrier. Caracterización Geomorfológica como Propuesta para la Planificación Ambiental y Territorial.

Un Ejemplo de Aplicación en Brasil con Perspectiva de Aplicación en Costa Rica

\section{Geomorfología y cartografía geomorfológica}

Mescerjakov (1968) establece la clasificación del relieve para el análisis geomorfológico en dos categorías principales: morfoestructura y morfoescultura. La morfoestructura se presenta con diferentes orígenes y edades, en esta clasificación se encuentran los cinturones orogénicos, las depresiones denudacionales, las depresiones tectónicas y las cuencas sedimentarias. Las morfoesculturas conciernen a fases del relieve generadas sobre las morfoestructuras, a través de procesos exógenos, es decir, el relieve es modelado por procesos ambientales que modifican las morfoestructuras, formando sobre ellas las morfoesculturas como las mesetas, las sierras, los cerros, entre otros. Por lo tanto, es una visión geomorfológica con fuerte enfoque geológico.

Ross (1992) propone una metodología para la cartografía geomorfológica, en donde establece dos niveles jerárquicos de clasificación del relieve: un primer taxón o mayor, corresponde a la morfoestructura y un segundo taxón o menor, son las unidades morfoesculturales, generadas a partir de la acción climática y la tectónica reciente a lo largo del tiempo geológico.

Para esta representación cartográfica cada unidad morfoestructural se representa con un color, y cada tonalidad de este color establece una unidad morfoescultural (Figura 2). Cada unidad morfoestructural presenta diferentes formas menores del relieve que difieren en función de su rugosidad topográfica, el índice de disección del relieve, su morfometría, e inclinación de las pendientes.

Se presenta un tercer taxón, dentro de la unidad morfoestructural y de dimensión inferior denominado Patrones de Formas del Relieve (Figura 2), este taxón hace referencia a modificaciones por procesos morfodinámicos actuales que pueden ser percibidos. Estos Patrones de Formas de Relieve pueden ser: formas de acumulación como planicies fluviales o marinas, o formas producidas por procesos denudacionales y tectónicos recientes, como, colinas, mesetas, salto de fallas, entre otros. 
Tabla 1. Patrones de Formas del Relieve

\begin{tabular}{|l|l|}
\hline \multicolumn{1}{|c|}{ FORMAS DE DENUDACIÓN } & \multicolumn{1}{c|}{ FORMAS DE ACUMULACIÓN } \\
\hline D - DENUDACIÓN & A - ACUMULACIÓN \\
Da - Formas agudas & Apf - Formas de planicie fluvial \\
Dc - Formas convexas & Apm - Formas de planicie marina \\
Dm - Formas en mesetas & Apl - Formas de planicie lacustre \\
Dp - Formas de superficies planas & Api - Formas de planície intermareal (manglar) \\
De - Formas de escarpes & Ad - Formas de campos de dunas \\
& Atf - Formas de terrazas fluviales \\
& Atm - Formas de terrazas marinas \\
\hline
\end{tabular}

Fuente: Ross (1992)

Para las formas de acumulación, se incorporaron nuevos términos que se agregan a los propuestos por Ross (1992) como formas de acumulación coluvial. Estas adaptaciones fueron necesarias, debido a la escala de trabajo utilizada, puesto que en algunos casos no fue posible separar terrazas fluviales de planicies fluviales, terrazas marinas de planicies marinas, depósitos coluviales de terrazas fluviales. Por lo tanto, se adoptaron nuevas clases de formas: terrazas y planicies marinas (Atpm), formas de depósitos coluviales, terrazas y planicies fluviales (Actpf), formas de depósitos coluviales y terrazas fluviales (Actf). Los patrones de forma de relieve se clasifican de acuerdo con la siguiente matriz, que relaciona en las columnas el grado de incisión de los valles y en las filas la dimensión interfluvial media (Figura 3).

Tabla 2. Matriz de índices de disección del relieve

\begin{tabular}{|c|c|c|c|c|c|}
\hline $\begin{array}{c}\text { DIMENSIÓN } \\
\text { INTERFLUVIAL MEDIA } \\
\text { (clases) } \\
\text { INCISIÓN MEDIA } \\
\text { DE LOS VALLES (clases) }\end{array}$ & $\begin{array}{c}\text { Muy } \\
\text { Grande } \\
(1) \\
>1500 \mathrm{~m}\end{array}$ & $\begin{array}{c}\text { Grande } \\
(2) \\
1500 \text { a } 700 \\
m\end{array}$ & $\begin{array}{c}\text { Media } \\
\text { (3) } \\
700 \text { a } 300 \\
\text { m }\end{array}$ & $\begin{array}{c}\text { Pequeña } \\
(4) \\
300 \text { a100 } \\
\text { m }\end{array}$ & $\begin{array}{c}\text { Muy } \\
\text { Pequeña } \\
(5) \\
<100 \mathrm{~m}\end{array}$ \\
\hline Muy Débil (1) (<20 m) & 11 & 12 & 13 & 14 & 15 \\
\hline Débil (2) (20 a $40 \mathrm{~m})$ & 21 & 22 & 23 & 24 & 25 \\
\hline Medio (3) (40 a $80 \mathrm{~m})$ & 31 & 32 & 33 & 34 & 35 \\
\hline Fuerte (4) $(80$ a $160 \mathrm{~m})$ & 41 & 42 & 43 & 44 & 45 \\
\hline MuyFuerte (5) (> $160 \mathrm{~m})$ & 51 & 52 & 53 & 54 & 55 \\
\hline
\end{tabular}

Fuente: Modificado de Ross (1992) 
Max Furrier. Caracterización Geomorfológica como Propuesta para la Planificación Ambiental y Territorial. Un Ejemplo de Aplicación en Brasil con Perspectiva de Aplicación en Costa Rica

El cuarto taxón se refiere a los tipos de forma del relieve individuales dentro de los Patrones de Forma del Relieve. Estos tipos de forma del relieve pueden ser el resultado de procesos denudacionales, entre éstos, se encuentran las colinas, las lomas, los cerros aislados, y los depósitos de acumulación, como las planicies lacustres, fluviales y marinas aisladas. Este nivel jerárquico tiene similitudes entre sí, tanto en la morfología y morfometría, es decir, en la forma y el tamaño.

El quinto taxón, corresponde a las pendientes o sectores de pendientes que componen los tipos de formas de relieves individuales (Figura 4). Una pendiente o un mismo sector de pendiente es individual por las características geométricas, genéticas y dinámicas; este taxón puede ser representado mediante cartografía a escalas mayores que 1:25.000.

El sexto taxón, en orden descendente, son las formas más pequeñas identificables. Son formas producidas por la erosión y deposición actuales. Como ejemplo de este taxón, se encuentran las formas producidas por agentes naturales como las hondonadas, las cárcavas, cicatrices de movimientos de masas, las barras de arenas actuales y las formas antropogénicas, tales como: corte, rellenos sanitarios, excavaciones y minería, entre otros. (Figura 4). Estos dos últimos taxones no fueron mapeados en este trabajo, debido a que fue necesaria una reducción de la escala para adecuarla a la revista. 
Figura 2. Unidades taxonómicas de clasificación del relieve

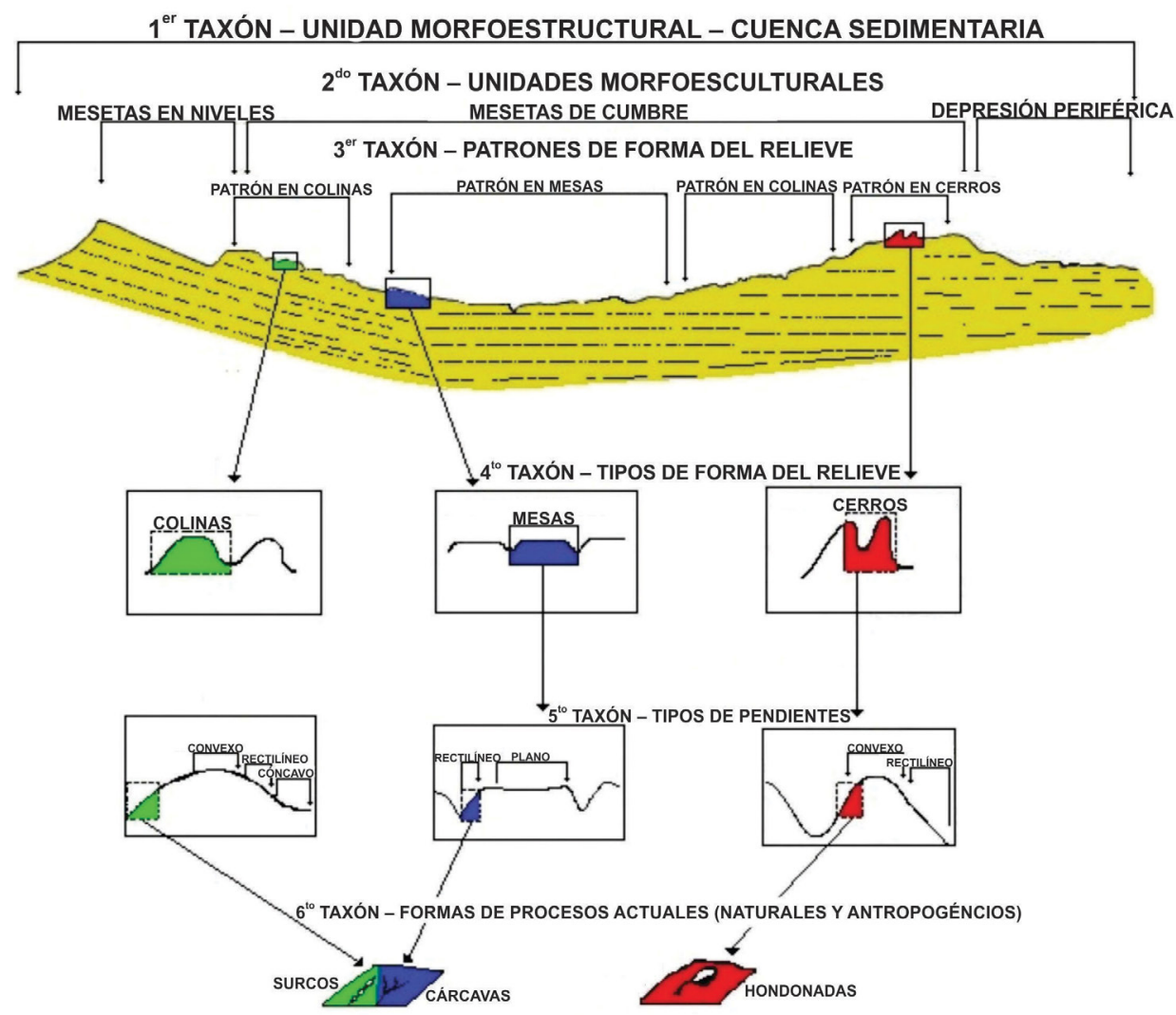

Fuente: Modificado de Ross (1992).

\section{Interacciones antrópicas en el medio ambiente}

Tomando la propuesta elaborada por Tricart (1977) e incrementado algunos criterios y parámetros, Ross (1994) elabora una nueva propuesta de análisis empírico de fragilidad de ambientes naturales y antrópicos. Cuando se implementa esta metodología, se debe tener especial preocupación por su aplicación en la planificación ambiental y territorial, partiendo del principio de que los recursos naturales deben ser utilizados por el hombre de forma racional, siempre respetando sus límites.

Ross (1994) elabora dos modelos empíricos para la evaluación de fragilidad de ambientes naturales y antrópicos, y los denomina respectivamente Unidades Ecodinámicas de Inestabilidad Potencial y Unidades 
Max Furrier. Caracterización Geomorfológica como Propuesta para la Planificación Ambiental y Territorial. Un Ejemplo de Aplicación en Brasil con Perspectiva de Aplicación en Costa Rica

Ecodinámicas de Inestabilidad Emergente. Las primeras pueden encontrarse en equilibrio y no han sufrido intervención humana, pero pueden entrar en desequilibrio por eventos naturales. Por el contrario, las segundas pueden verse afectadas por intervenciones antrópicas y ser modificadas intensamente.

Para jerarquizar tanto las Unidades Ecodinámicas de Inestabilidad Potencial (UEIP) como las Unidades Ecodinámicas de Inestabilidad Emergente (UEIE), son analizados de forma individualizada cuatro componentes: relieve, suelo, uso y ocupación de la tierra y clima. A cada componente se le asigna un valor que varía de 1 a 5 . Estos valores corresponden a los grados de fragilidad de cada componente en comparación con los eventos naturales y las intervenciones humanas.

Como producto se obtienen análisis individualizados de los componentes: relieve, suelo, uso y ocupación de la tierra y clima, con sus respectivas cartas temáticas y datos colectados en campo. A continuación, se realiza la superposición de los datos, obteniendo así la carta de fragilidad de los ambientes naturales y antrópicos. La superposición de las cartas temáticas generará áreas con un valor numérico de cuatro dígitos. Tomando como ejemplo un área cuyo conjunto numérico generado fue 1111, se podrá representar un relieve de disección muy débil (1), Latosol con textura arcillosa (1), cobertura forestal natural (1) y clima con precipitación anual baja y homogénea (1), características que corresponden a una Unidad Ecodinámica de Inestabilidad Potencial muy baja.

Además de ser representada por el conjunto numérico 111, es dada a las Unidades Ecodinámicas de Inestabilidad Potencial las variables del color verde. O sea, verde claro para UEIP alta y verde oscuro para UEIP baja. Para las UEIE es dada las variables de los colores rojo e naranja, que internacionalmente son asociadas a áreas de riesgos. Las UEIE muy altas reciben el tono rojo oscuro y las UEIE el tono naranja claro.

\section{Procedimientos técnicos y operacionales}

El área de estudio comprende dos cartas topográficas a escala 1:25.000, con equidistancia entre las curvas de nivel de $10 \mathrm{~m}$, publicadas por la SUDENE, en 1974 (Brasil, 1974). Estas cartas topográficas son las siguientes: carta Jacumã y carta Conde. Se optó por trabajar con cartas topográficas, debido su precisión, ya que fueron elaboradas por el método 
de aerotriangulación, que no es más utilizado debido a los altos costos, y poseen una equidistancia entre las curvas de nivel de $10 \mathrm{~m}$.

En una primera fase, se vectorizó todo el contenido de las cartas, principalmente las curvas de nivel para obtener la cartografía final digital con las informaciones necesarias para producir las cartas temáticas básicas, que fueron utilizadas para la elaboración de la plancha geomorfológica, tomando como base la metodología desarrollada por Ross (1992) con adaptaciones de Furrier (2007), y la plancha de Fragilidad de los Ambientes Naturales y Antrópicos (Ross, 1994). Fue utilizado el software libre Spring 5.1.2 desarrollado por el Intituto Nacional de Pesquisas Espaciais - INPE.

La carta geomorfológica fue elaborada con base en la metodología desarrollada por Ross (1992) y con algunas adaptaciones ya mencionadas. La carta de fragilidad de ambientes naturales y antrópicos fue elaborada siguiendo la metodología desarrollada por Ross (1994), adicionando a ella comportamientos pluviométricos definidos por Spörl (2001) y modificaciones metodológicas propuestas por Furrier (2007).

Su elaboración constituyó una sobreposición de informaciones extraídas de la carta geomorfológica, análisis e interpretación de imágenes satelitales para el uso y ocupación de la tierra y datos pedológicos extraídos de Paraíba (2004). Adicionalmente, se incorporaron datos climáticos. Estas informaciones fueron agrupadas en una leyenda integrada, que da soporte a la interpretación de la carta de fragilidad de los ambientes naturales y antrópicos. Así, esta carta es un documento síntesis de la investigación y junto a su leyenda, un excelente banco de datos.

\section{Resultados y discusiones}

La configuración del relieve del área expone una disposición de la red de drenaje muy vinculada a la morfoestructura generada por la tectónica reciente (Figura 3). A través de la plancha geomorfológica se observan ríos con sentido norte, cambiando completamente la ya conocida inclinación de la Formación Barreiras en dirección al océano. Esta morfología de la red de drenaje observada en el compartimiento Dm 42, corrobora junto a los estudios recientes, que la tectónica ejerce un papel fundamental en la morfología del relieve brasileño, cambiando conceptos arraigados en el desarrollo del relieve sólo por factores climáticos en la margen continental pasiva. 
Además del sentido irregular de los ríos y arroyos, se visualizan fuertes inflexiones en ríos como el Río Graú (Dsc 43/Dm 42) y Río Gurují (Dm42). En este último río ocurre una inflexión fuerte de $90^{\circ}$ a $230 \mathrm{~m}$ de la línea de costa, ocasionada por una falla normal que obstruye el río y expone a la Formación María Farinha en la playa de Jacumã en la superficie en un nivel de $5 \mathrm{~m}$ (Figura 3).

Figura 3. Plancha geomorfológica del área de estudio.

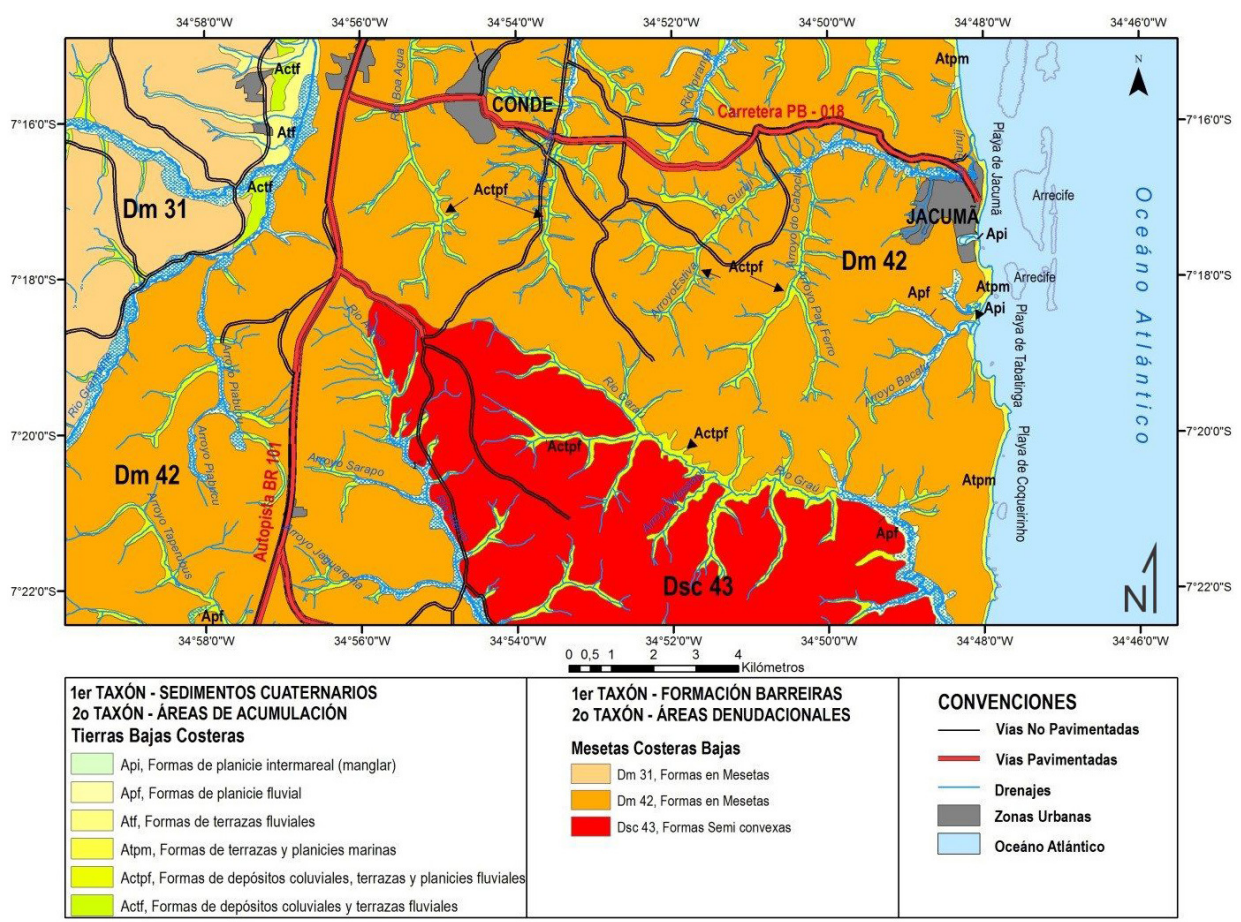

Fuente: Elaboración propia.

El Río Aterro está ubicado sobre la línea de falla homónima y su carácter rectilíneo es nítidamente visible. Este río separa dos compartimientos del relieve bien distintos, uno en el que se encuentran la altimetría e inclinación de las pendientes más fuertes (Dsc 43) y otro con forma típica de mesetas (Dm 42), cada uno con su configuración y densidad de la red de drenaje dispareja (Figura 3). 
También en contra de la mencionada inclinación de la Formación Barreiras hacia el océano, al oeste del área se encuentra el compartimiento Dm 31, en donde las mesetas son más amplias, pero con las altimetrías más bajas. Este compartimiento se encuentra separado de forma brusca del compartimiento Dm 42 por el Río Gramame, el cual, dentro el área de estudio, fluye hacia el norte, pero después de salir del área sufre una fuerte inflexión de $90^{\circ}$ y cambia su sentido hacia el este, en dirección al océano.

La configuración geomorfológica del área tiene una fuerte influencia en la distribución de los diversos usos de la tierra verificados. Las mesetas más amplias y aplanadas encontradas en la parte oeste están prácticamente ocupadas por los cultivos industriales de caña de azúcar, en un sistema intensamente mecanizado, competitivo y fundamental para el mercado capitalista actual.

La urbanización avanza fuertemente sobre las mesetas que proporcionan vista al mar, sobre las cuales se construyen grandes condominios cerrados de estrato socioeconómico alto. Sobre manglares, planicies fluviales y mesetas interiores avanza una urbanización de estrato socioeconómico más bajo. Pequeñas y medianas propiedades rurales, con cultivos temporales y/o perennes, están asentados sobre los compartimientos de relieve más disectados, donde es difícil la intensificación de la mecanización agrícola.

Después de la caracterización de los diversos componentes del medio físico, del uso y ocupación de la tierra, que es un componente humano y económico, y de la síntesis de varias informaciones, fue elaborada la plancha de Fragilidad de los Ambientes Naturales y Antrópicos (Figura 4). Está plancha tiene con base de apoyo principal la plancha geomorfológica elaborada aquí.

Observando la plancha de fragilidad, se visualizan con claridad, las clases verificadas con mayores grados de fragilidad potencial y emergente, de acuerdo con el cruce de información de relieve (1), suelos (2), uso y ocupación de la tierra (3) y el comportamiento pluviométrico (4). La secuencia numérica de componentes naturales sigue este orden en la plancha de fragilidad, y con el apoyo de las tablas integradas al mismo (leyenda), la comprensión se facilita, incluso teniendo un gran número de información.

Las Unidades Ecodinámicas de Instabilidad Potencial (tonos verdes) son verificadas en zonas con vegetación natural o en estado de elevada 
Max Furrier. Caracterización Geomorfológica como Propuesta para la Planificación Ambiental y Territorial.

Un Ejemplo de Aplicación en Brasil con Perspectiva de Aplicación en Costa Rica

regeneración, y están muy restringidas en el área, ocupando pendientes con elevadas inclinaciones, en donde se dificulta la agricultura o en reservas naturales como la Área de Protección Ambiental de Tambaba (APA - Tambaba), en el sur del área. Al cruzar toda la información se obtuvo un conjunto numérico 4412 en su sector norte y 4423 en su sector sur, la diferencia entre los dos sectores se debe a la regeneración de la vegetación natural, siendo está más expresiva en el sector norte (Figura 4). 
Max Furrier. A Geomorphological Characterization as a Proposal for Environmental and Territorial Planning. An Example of Application in Brazil Intended to Be Replicated in Costa Rica

Figura 4. Plancha de fragilidad de los ambientes naturales y antrópicos, con los cuadros de los valores (1-Relieve, 2- Suelos, 3- Uso y ocupación de la tierra, 4- Comportamiento Pluviométrico).

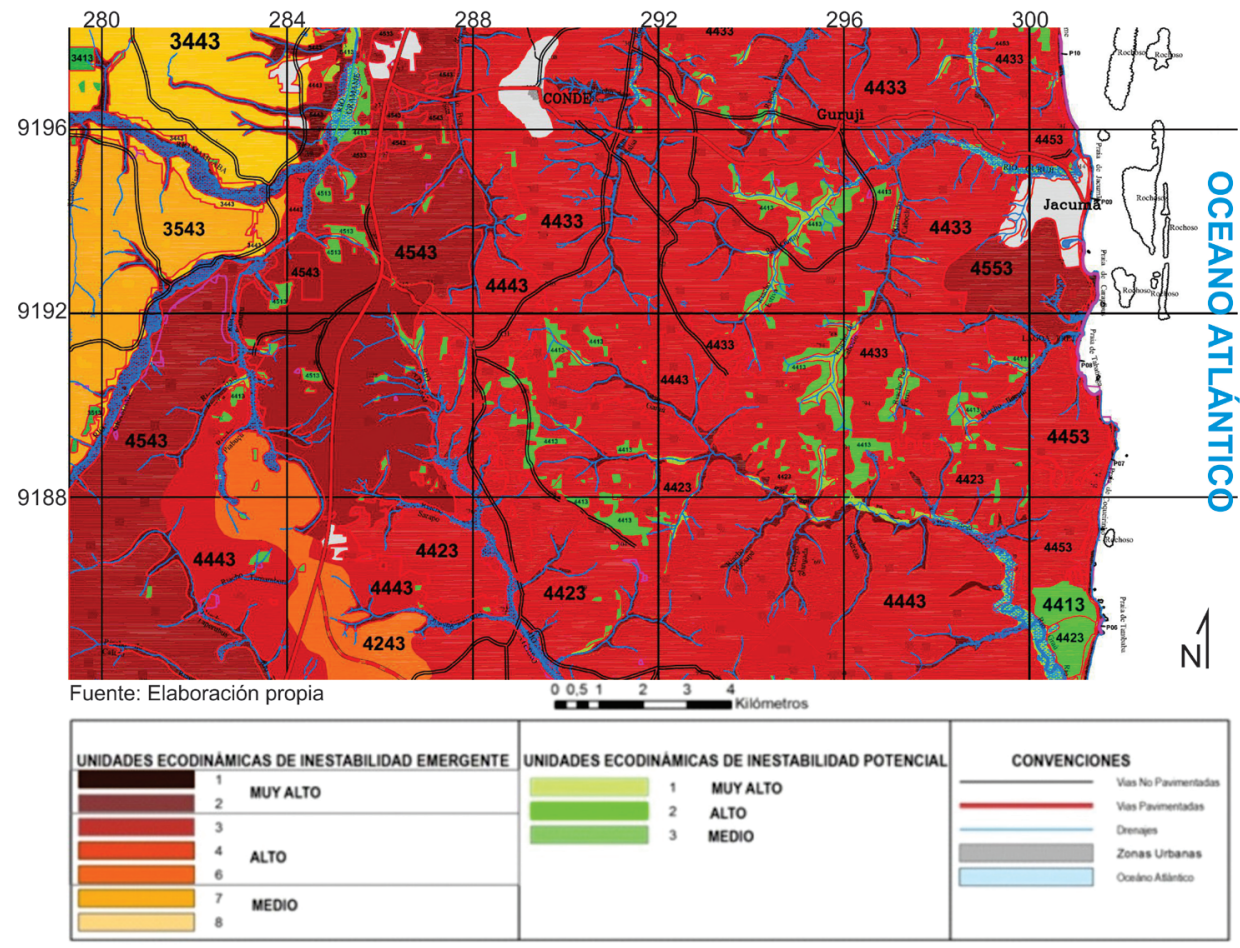

1 CLASES DE FRAGILIDAD BASADAS EN LOS TIPOS DE FORMAS
DEL RELIEVE ENCONTRADOS EN EL ÁREA DE ESTUDIO
\begin{tabular}{|l|l|}
\hline GRADOS DE FRAGILIDAD & TIPOS DE FORMA DEL RELIEVE \\
\hline $1-$ MUY DÉBIL & Sin ocurrencia en el área \\
\hline $2-$ DÉBIL & Dm 21 \\
\hline $3-$ MEDIO & Dm 31. Dm 32. Dm 33. Dm 13. Dm 23 \\
\hline $4-$ FUERTE & Dm 41. Dm 42. Dsc 43. Dc 24. Atf. Actf \\
\hline $5-$ MUY FUERTE & Api. Apf. Atpm. Actpf \\
\hline Fuente: Ross (1994)
\end{tabular}

3 CLASES DE PROTECCIÓN POR TIPOS DE USO Y OCUPACIÓN DE LA TIERRA ENCONTRADOS EN EL ÁREA DE ESTUDIO \begin{tabular}{|l|l|l|}
\hline GRADOS DE PROTECCIÓN & TIPOS DE COBERTURA VEGETAL \\
\hline $1-$ MUY ALTA
\end{tabular} \begin{tabular}{|l|l}
\hline $1-$ MUY ALTA & Bosques naturales y/o en avanzado \\
\hline
\end{tabular} \begin{tabular}{|l|l|} 
& estado de reqeneración. Manalares. \\
\hline 2 -ALTA & $\begin{array}{l}\text { Formaciones arbustivas naturales con } \\
\text { estrato herbáceo denso (cerrado). } \\
\text { Formaciones arbustivas densas }\end{array}$ \\
\hline
\end{tabular} \begin{tabular}{l|l} 
estrato herbáceo denso (cerrado). \\
Formaciones arbustivas densas \\
(bosques secundarios). Cultivos de \\
ciclos largos como el bambú, campos \\
halófilos, hiarófilos e hidrófilos.
\end{tabular} \begin{tabular}{l|l}
\hline $3-$ MÉDIA & $\begin{array}{l}\text { Cultivos temporales y cultivos } \\
\text { permanentes cuando no pueden ser }\end{array}$
\end{tabular} 4-BAJA $\quad$ separados debido a la escala adoptada \begin{tabular}{l|l}
\hline $4-B A J A$ & Caña-de-azúcar y cultivos temporales
\end{tabular} \begin{tabular}{l|l}
\hline $5-$ MUY BAJA & Cortos como alaodon. \\
\hline & Suelo expuesto y lotes sin
\end{tabular} Fuente: Ross (1994) infraestructura urbana.
2 CLASES DE FRAGILIDADES DE LOS TIPOS DE SUELOS ENCONTRADOS EN EL ARE DE ESTUDIO EN RELACIÓN CON LA EROSIBILIDAD E INUNDACIÓN

\begin{tabular}{|l|l|}
\hline CLASES DE FRAGILIDAD & TIPOS DE SUELOS \\
\hline
\end{tabular}

\begin{tabular}{|l|l|}
\hline \multicolumn{1}{|c|}{ CLASES DE FRAGILIDAD } & \multicolumn{1}{c|}{ TIPOS DE SUELOS } \\
\hline 1-MUY BAJA & Sin ocurrencia en el área de estudio. \\
\hline 2-BAJA & Latosoles roios-amarillos distróficos. \\
\hline 3-MEDIA & $\begin{array}{l}\text { Argisolos rojo-amarillo álico, Argisolos } \\
\text { rojo-amarillo distrófico, Argisolos rojo- } \\
\text { amarillo eutrófico. }\end{array}$ \\
\hline 4-ALTA & $\begin{array}{l}\text { Argisolos rojo-amarillo álico (textura } \\
\text { grosera), Argisolos rojo-amarillo } \\
\text { distrófico (textura grosera), Argisolos } \\
\text { rojo-amarillo eutrófico (textura grosera), } \\
\text { Suelos aluviales eutróficos. }\end{array}$ \\
\hline $5-$ MUY ALTA & $\begin{array}{l}\text { Espodosuelos hidromórficos, Gleisuelos } \\
\text { distróficos indiscriminados, suelos } \\
\text { indiscriminados de manglar, Neosuelos } \\
\text { quartzarénicos. }\end{array}$ \\
\hline
\end{tabular}

Fuente: Ross (1994)

4 COMPORTAMENTO PLUVIOMÉTRICO DEL AREA DE ESTUDIO GRADOS DE IMPACTO COMPORTAMENTO

\begin{tabular}{l|l}
\hline $3-\mathrm{MÉDIO}$ & Situación pluviométrica con distribución
\end{tabular} entre 2 a 3 meses. Pluviosidad media Fuente: Spörl (2001) $1.700 \mathrm{~mm}$. 
Max Furrier. Caracterización Geomorfológica como Propuesta para la Planificación Ambiental y Territorial. Un Ejemplo de Aplicación en Brasil con Perspectiva de Aplicación en Costa Rica

Como se puede observar en la plancha de fragilidad, existe un gran predominio de áreas para la Unidades Ecodinámicas de Inestabilidad Emergente (tonos rojos y naranjas) frente a la fuerte actuación antrópica. Al sur del casco urbano de Jacumã fueron verificadas unidades de inestabilidad emergente más fuertes, con un conjunto numérico 4553. Esta área se ubica sobre un relieve Dm 42, Espodosuelos Hidromórficos asociados a Latosoles rojos-amarillentos, suelos expuestos por el avance de la urbanización sin planificación y el comportamiento pluviométrico medio de $1.700 \mathrm{~mm} / a n ̃ o$. Cabe resaltar que, debido a la dimensión reducida del área, alrededor $296 \mathrm{~km}^{2}$, solo se verifica un comportamiento pluviométrico de nivel medio en la escala propuesta por Spörl (2001).

Por lo tanto, observando la plancha de fragilidad con su leyenda integrada, se percibe una enorme gama de información de uso práctico, con cada algoritmo asociado a un componente natural o a un tipo de uso de la tierra empleado. Es decir, esta plancha es una síntesis de todo el levantamiento de los componentes naturales y antrópicos verificados. La clasificación de cada componente en su grado de fragilidad es basada en estudios empíricos y el conocimiento general.

Utilizando el software libre Spring 5.4.1, fue posible cuantificar el área de cada nivel de inestabilidad potencial e inestabilidad emergente, generando datos concretos sobre cuáles son los niveles más encontrados. Añadiendo las informaciones de la plancha de fragilidad, se obtiene información de utilización práctica, tanto para uso en el ordenamiento territorial como en la planificación ambiental (Figura 5). 
Figura 5. Porcentaje de los niveles de inestabilidades emergente y potencial encontradas en el área de estudio.

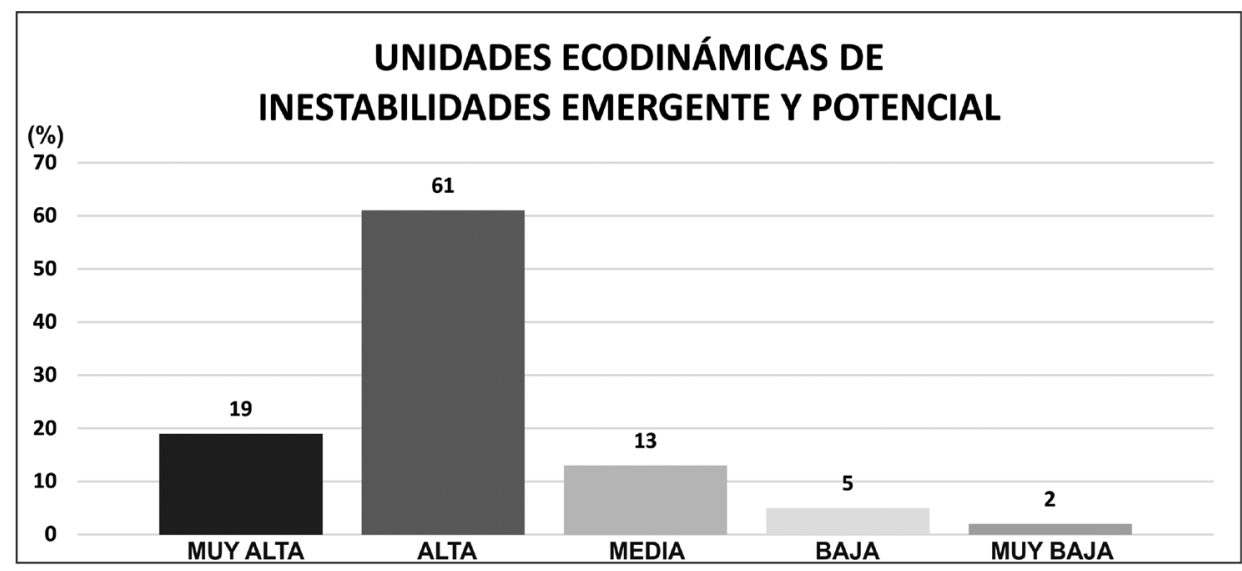

Fuente: Elaboración propia.

\section{Consideraciones generales}

La propuesta central del presente trabajo fue presentar una metodología basada en el mapeo geomorfológico y elaborar una caracterización que involucrara una serie de levantamientos cartográficos, así como la búsqueda de datos, muchas veces dispersos en servicios geológicos, geográficos, institutos agrícolas y ambientales. Al mismo tiempo, se percibe que una caracterización total y plena es una tarea imposible; no obstante, a partir de la integración de los datos obtenidos, generados, analizados y discutidos resultó una visión completa del área, en la que es posible visualizar el valor que toman los componentes del medio físico que interactúan, y cómo esa interacción influye en las características socioeconómicas y su espacialización.

La elaboración de una plancha geomorfológica, basada tanto en la morfoestructura como en la morfoescultura, no sólo es un importante instrumento de caracterización del relieve, sino que adicionalmente puede apoyar otras caracterizaciones ulteriores, como suelo, uso y ocupación de la tierra. Además, representa una síntesis geomorfológica descrita, así como un valioso instrumento para investigaciones futuras, tanto en el campo de la geomorfología como en las áreas relacionadas, y finalmente, se presenta como una importante herramienta en estudios ambientales para 
la planeación del territorio, planeación ambiental y planes municipales. El relieve es el escenario de todas las actividades sociales.

La caracterización de los componentes del medio físico, con un gran enfoque en la geomorfología desarrollada en este estudio, demuestran qué tan necesario es pensar en el todo y de qué modo ese todo se manifiesta en la realidad y se integra con ella. La comprensión parcial de esta realidad, sin tener una visión global o de conjunto, puede producir resultados insatisfactorios. Así, es necesario para los geomorfólogos, en general, una buena base de datos y conocimientos geológicos, ya que el relieve está sostenido por una geología, muchas veces complexa y desconocida.

La plancha de Fragilidad de los Ambientes Naturales y Antrópicos, con los cuadros de los valores (1-Relieve, 2- Suelos, 3- Uso y ocupación de la tierra, 4- Comportamiento Pluviométrico) muestra ser un documento sintético con las informaciones espaciales y condensadas en un único producto cartográfico. Por lo tanto, se puede decir que es un documento apto para la utilización en la planificación territorial y ambiental, además de ser de extrema utilidad para el ordenamiento territorial, pues indica donde hay áreas con unidades con mayores y menores inestabilidades.

El trabajo terminado demuestra también la cantidad de información que puede ser extraída, obtenida y generada con un mínimo de recursos técnicos posibles, pero con un desarrollo científico adecuado que reúne las diferentes especializaciones que surgen dentro de las más variadas áreas del conocimiento, y pone de manifiesto que una caracterización básica de los componentes de un área geográfica puede ser extremadamente útil a la ciencia y la sociedad. Países en desarrollo como Brasil y Costa Rica deben conocer más sus aspectos naturales de manera holística e integrada, así, esta metodología aquí demostrada podrá, con los ajustes necesarios, ser aplicada también en regiones costeras de Costa Rica. 


\section{Referencias}

Ab'Saber, A. N. (2000). Fundamentos da geomorfologia costeira do Brasil atlântico inter e subtropical. Revista Brasileira de Geomorfologia, $1(1), 27-43$.

Brasil. Instituto Brasileiro de Geografia e Estatística (2016). Estimativa Populacional 2016. Consultado el 25 de mayo de 2016.

Brasil. Superientendêndia de Desenvolvimento do Nordeste. (1990). Dados pluviométricos mensais do Nordeste - Estado da Paraíba. Recife: SUDENE.

Brasil. Ministério do Interior. (1974). Superintendência de Desenvolvimento do Nordeste. Folhas , Jacumã, Conde, Recife: SUDENE. Escala 1: 25.000 .

Brito Neves, B., Riccomini, C., Fernandes, T. M. G. y Sant'Anna, L. G. O. (2004). Sistema tafrogênico terciário do saliente oriental nordestino na Paraíba: um legado proterozoico. Revista Brasileira de Geociências, 34(1), 127-134.

Feitosa, A. C. (1996). Dinâmica dos processos geomorfológicos da área costeira a nordeste da ilha do Maranhão, 249f. (Tese Doutorado Instituto de Geociências e Ciências Exatas, UNESP) Rio Claro.

Françolin, J. B. L. y Szatmari, P. (1987).Mecanismo de rifteamento da porção oriental da margem norte brasileira, Revista Brasileira de Geociências, 17(2), 196-207.

Furrier, M. (2007). Caracterização geomorfológica e do meio físico da Folha João Pessoa-1:100.000. 213f. (Teses Doutorado em Geografia Física. Departamento de Geografia, Universidade de São Paulo). São Paulo.

Hoefel, F. G. (1998). Morfodinâmica de Praias Arenosas Oceânicas: uma Revisão Bibliográfica. Editora da Universidade do Vale do Itajaí, Itajaí.

Leal e Sá (1998). Levantamento geológico-geomorfológico da Bacia Pernambuco-Paraíba, no trecho compreendido entre Recife-PE e João Pessoa-PB. 127f. Dissertação (Mestrado) - Centro de Tecnologia, Universidade Federal de Pernambuco, Recife.

Lepsch, I. F. (2002). Formação e conservação dos solos. São Paulo: Oficina de Texto.

Libault, A. (1971). "Os quatro níveis da pesquisa geográfica” Métodos em questão. São Paulo: IGEOG-USP, (1), 1-14. 
Max Furrier. Caracterización Geomorfológica como Propuesta para la Planificación Ambiental y Territorial.

Un Ejemplo de Aplicación en Brasil con Perspectiva de Aplicación en Costa Rica

Marques, J. S. (1994). Ciência geomorfológica. En: Teixeira A. J. y Cunha S. B. (Org.), Geomorfologia uma atualização de bases e conceitos. Bertrand Brasil, Rio de Janeiro, pp. 23-50.

Mescerjakov, J. P. (1968). "Les concepts de morphostructure et de morphosculpture: unnouvelinstrument de l'analyse géomorphologique". Annales de Géographie, 77, (423), 539-552

Mesquita, A. R. (2003). Sea-level variations along the Brazilian coast: a short review. Journal of Coastal Research, S.I. (35), 21-31.

Moreira, E. y Targino, I. (1997). Capítulos de geografia agrária da Paraí$b a$. João Pessoa: EDUFPB.

Muehe, D. (1994). Geomorfologia Costeira. In: Guerra, A. J. T.; Cunha, S. B. Geomorfologia: Uma atualização de bases e conceitos. Rio de Janeiro: Bertrand.

Paraíba. SUDEMA. (2004). Mapa pedológico do Estado da Paraíba, João Pessoa, SUDEMA. Escala: 1:500.000. 1 mapa color.

Ross, J. L. S. (1990). Geomorfologia: Ambiente e Planejamento. Contexto.

Ross, J. L. S. (1992). O registro cartográfico dos fatos geomórficos e a questão da taxonomia do relevo, Revista do Departamento de Geografia, 6, 17-29.

Ross, J. L. S. (1994). Análise empírica da fragilidade dos ambientes naturais e antropizados. Revista do Departamento de Geografia da FFLCH/USP, São Paulo, 8, Departamento de Geografia, USP, Brasil. 63-74.

Ross, J. L. S. (1996). Geomorfologia aplicada aos EIAs-RIMAs. En: Teixeira Guerra, A. J. y Cunha, S. B. (Org.). Geomorfologia e meio ambiente, (291-336). Bertrand Brasil, Rio de Janeiro.

Slocombe, D. S. (1993). "Environmental planning, ecosystem Science, and ecosystem approaches for integrating and development". Environmental Management, 17, 289-303.

Santos, R. F. (2004). Planejamento Ambiental: teoria e prática. Oficina de Texto.

Spörl, C. (2001). Análise da fragilidade ambiental relevo-solo com aplicação de três modelos alternativos nas altas baciais do rio Jaguari-Mirim, ribeirão do Quartel e ribeirão da Prata. 160f. Dissertação (Mestrado) - Faculdade de Filosofia, Letras e Ciências Humanas, Universidade de São Paulo, São Paulo. 
Max Furrier. A Geomorphological Characterization as a Proposal for Environmental and Territorial Planning. An Example of Application in Brazil Intended to Be Replicated in Costa Rica

Suguio, K.; Angulo, R. J.; Carvalho, A. M.; Corrêa, I. C. S.; Tomazelli, L. J.; Willwock, J. A. Vital, H. (2005). Paleoníveis do mar e paleolinhas de costa. En: Souza, C. R.; Suguio, K.; Oliveira, A. M. S.; DE Oliveira, P. E. (Ed.). Quaternário do Brasil. (114-129). Ribeirão Preto: Holos,Editora.

Tricart, J. (1977). Ecodinâmica.Rio de Janeiro: FIBGE. 